\title{
Editorial
}

\section{Más allá de la vacuna, la mejor estrategia contra la COVID-19 es mantener a la población saludable}

Quiero hacer algunos comentarios al artículo "Cambios en el peso corporal, la actividad física y el estilo de vida durante el confinamiento obligatorio por COVID-19 en Colombia: una encuesta en línea", publicado por Rosero-Olarte, Pantoja, Builes-Barrera y Ibarra-Jurado en la presente edición (Vol. 8, No. 2, 2021) y en la cual se recolectaron 1434 encuestas online a personas adultas, para evaluar el comportamiento del peso y su relación con hábitos y estilos de vida saludables (HEVS), en especial a lo referente a la alimentación y la actividad física. Los resultados principales fueron que el $28,6 \%$ de los encuestados reportaron un aumento de peso corporal, el $29,1 \%$ reportó disminución de peso y el $42,3 \%$ se mantuvo igual; además, el $49,7 \%$ reportó una mejoría en sus hábitos de alimentación y el $18,8 \%$ empeoró y en lo que respecta a hábitos en la actividad física, el 52,3\% informó haber estado realizando algún tipo de actividad física durante el periodo de confinamiento.

Al cruzar la información, los investigadores encontraron que las personas que disminuyeron de peso, versus aquellas que aumentaron de peso, reportaron tener mejores hábitos de alimentación ( $80,8 \%$ vs. $24,9 \%$ ( $p<$ $0,001)$, y de actividad física $(71,2 \%$ vs. $26,8 \%(p<0,001)$.
Además de las limitaciones y las falencias del estudio, expresados por los mismos autores, me quedan otras dos preocupaciones: la primera es que las encuestas fueron iniciadas el 1 de abril, es decir, solo ocho días después de la orden de encierro por cuarentena $y$, por lo tanto, para las primeras encuestas se llevaban muy pocos días de encierro como para sacar conclusiones veraces, y la segunda es que los resultados no se discriminaron por estado de peso antes del encierro, en tanto que los obesos probablemente se comportarán de manera distinta a los con peso normal.

Al leer este artículo y otros similares podría inferir que existen tres tipos de pacientes: los primeros son los que se "echaron a la locha", a estar sedentarios y a comer más de la cuenta, en especial productos ultraprocesados y comida rápida, y probablemente también se dedicaron a dormir más tiempo y a gastar más horas frente a pantallas. Eventualmente, el perfil de estas personas no es nuevo, sino que se agravó durante el encierro y podría decirse que "ya eran así" y que muchos de ellos tenían sobrepeso u obesidad, pero que, finalmente, con el encierro aumentaron más de peso. Los segundos son los que aprovecharon para cocinar más saludable, dejaron de consumir comidas rápidas, generalmente hipercalóricas y 
se propusieron realizar más actividad física en casa. Probablemente estas personas, desde antes de la pandemia, ya se cuidaban para mantener un buen peso y probablemente no eran obesas, o si acaso tenían un ligero sobrepeso. Quizás muchos de ellos durante el encierro mantuvieron su peso o, incluso, algunos pudieron haber bajado de peso. Finalmente, el tercer grupo son los que continuaron su rutina de alimentación y de actividad física sin mayores cambios a como lo hacían antes del aislamiento. Estos finalmente con el encierro mantuvieron su peso sin mayor variación.

Por supuesto que la pandemia nos expuso a una vida potencialmente más sedentaria y con mayores posibilidades de cambiar la forma de alimentarnos, además de generarnos estados de ansiedad y depresión que magnifican estos cambios, pero la actitud y la forma de comportarnos también determinan los resultados (2). Esto lo explicaré con una anécdota, por estos días uno de mis pacientes me dijo: ¡Ay, doctor, por el confinamiento a causa de la pandemia he aumentado seis kilos! A esto le respondí: ¡Pues le cuento que yo, por lo contrario, he bajado seis kilos durante la pandemia! A lo que replicó: "pues vea que así es, pues uno de mis hijos que es deportista se ha puesto a hacer ejercicio en casa y si viera cómo anda de bien". En conclusión, se sube, se baja o se mantiene el peso, no solo por las causas externas que le favorezcan, sino por la actitud personal y por lo que cada uno quiere para sí mismo, independientemente de las condiciones externas.

Hasta la fecha se han publicado multitud de artículos y metaanálisis de estos mismos que hacen referencia a este tema, con conclusiones similares: el encierro por la pandemia ha hecho que se modifiquen los HEVS en la población y esto ha llevado a que algunos bajen, otros suban y otros se queden con el mismo peso de siempre, con diferentes porcentajes entre uno y otro estudio. Quizás, las circunstancias de cada reporte son diferentes, muy en relación con la edad y el sexo de los encuestados o evaluados, el IMC previo de ellos y la cercanía con el momento de la confinación. Es de anotar que, en general, los niños y los jóvenes son los que más han aumentado de peso y que las personas de mayor edad, por lo contrario, en mayor proporción han reducido o mantenido el mismo peso, más notablemente las mujeres $(2,3,4,5)$.

A manera de información básica, varias situaciones en el área de la nutrición, extraídas del Ensin 2010 (6), son determinantes para el alto porcentaje de sobrepeso y obesidad en Colombia. Ellas son: entre personas de 5 a 64 años, uno de cada tres no consumen frutas diariamente; cinco de cada siete no consumen hortalizas o verduras diariamente; uno de cada siete no come carnes o huevos diariamente; uno de cada dos consume embutidos de forma semanal; uno de cada cuatro consume comidas rápidas semanalmente; uno de cada dos consume gaseosas o refrescos semanalmente; cuatro de cada nueve consumen alimentos de paquete semanalmente; uno de cada tres consume golosinas y dulces diariamente y una cuarta parte de los colombianos consumen alimentos en la calle semanalmente. El artículo de Rosero et al. no hace la relación de los alimentos consumidos por los encuestados durante el encierro, pero sí señala que del grupo 
que aumentó peso, el 45,4 \% refirió tener peores hábitos de alimentación comparado contra $3,6 \%$ y $11,4 \%$ en los grupos de disminución de peso o sin cambios, respectivamente $(p<0,001)$.

Con referencia a la actividad física, el Ensin 2015 informa que solo el 51,3\% de los adultos colombianos cumplen con las recomendaciones de actividad física (7). De forma similar, en el artículo de Rosero et al, el 52,3\% de los encuestados autorreportaron haber estado realizando algún tipo de actividad física durante este periodo de confinamiento, pero cabe señalar que el grupo que dijo que aumentó de peso también reportó realizar menos actividad física.

La obesidad es uno de los mayores factores de riesgo para sufrir COVID-19 y para padecer mayor morbilidad y mortalidad por la misma. Por lo tanto, es muy preocupante el incremento de peso que se ha producido de manera global en toda la población durante la pandemia, favorecido por los malos hábitos de alimentación y el sedentarismo. También preocupa que no solo se evidenció un aumento de peso durante el tiempo del encierro, sino que también se documentó durante el periodo posterior al confinamiento. (8).

Señalo que la estrategia para mejorar los parámetros de morbilidad y mortalidad por la pandemia de COVID-19 no se producirá únicamente con la vacunación. Se deben mejorar las condiciones de salud de la población y propender por la promoción activa de los HEVS, en especial la actividad física y la alimentación saludable. Más allá de la vacunación, la pandemia actual nos ha enseñado la importancia de las medidas preventivas en el estilo de vida.
De hecho, un estilo de vida físicamente activo previene la inmunosenescencia a través de varios mecanismos. Los confinamientos, el distanciamiento, y la buena higiene son necesarios, pero también lo es la actividad física, que no solo es segura, sino que tiene un papel preventivo potencial, especialmente para los grupos más vulnerables (9).

Iván Darío Escobar Duque Instituto de Diabetes y Endocrinología Bogotá, Colombia Correo electrónico: ivan_escobard@yahoo.com

\section{Referencias}

[1] Rosero-Olarte FO, Pantoja D, Builes-Barrera CA, Ibarra-Jurado JC. Cambios en el peso corporal, la actividad física y el estilo de vida durante el confinamiento obligatorio por COVID-19 en Colombia: una encuesta en línea. Rev Col End. 2021; 8(2):e710. DOI: https://doi. org/10.53853/encr.8.2.710

[2] Chew HS, López V. Global Impact of COVID-19 on Weight and WeightRelated Behaviors in the Adult Population: A Scoping Review. Int J Environ Res Public Health. 2021;18(4):1876. DOI: https:// doi.org/10.3390/ijerph18041876

[3] Lange SJ, Kompaniyets L, Freedman DS, Kraus EM, Porter R, Blanck $\mathrm{HM}$, et al. Longitudinal Trends in Body Mass Index Before and During the COVID-19 Pandemic Among Persons Aged 2-19 Years 
- United States, 2018-2020. MMWR Morb Mortal Wkly Rep. 2021;70(37):1278-83. DOI: https://doi.org/10.15585/mmwr. mm7037a3

[4] Bakaloudi DR, Barazzoni R, Bischoff SC, Breda J, Wickramasinghe K, Chourdakis M. Impact of the first COVID-19 lockdown on body weight: A combined systematic review and a meta-analysis. Clin Nutr. 2021. DOI: https://doi. org/10.1016/j.clnu.2021.04.015

[5] He M, Xian Y, Lv X, He J, Ren Y. Changes in Body Weight, Physical Activity, and Lifestyle During the Semi-lockdown Period After the Outbreak of COVID-19 in China: An Online Survey. Dis Med Public Health Prep. 2021;15(2):e238. DOI: https://doi.org/10.1017/ dmp.2020.237

[6] Profamilia, Instituto Nacional de Salud, Instituto Colombiano de Bienestar Familiar. Encuesta Nacional de la Situación Nutricional en Colombia, ENSIN-2010 [Internet]. Disponible en: https:// www.icbf.gov.co/sites/default/ files/resumenfi.pdf

[7] Ministerio de Salud y Protección Social. Encuesta Nacional de la Situación Nutricional en Colombia ENSIN-2015 [Internet]. Disponible en: https://www.minsalud.gov.co/ sites/rid/Lists/BibliotecaDigital/ RIDE/VS / ED / G CFI/ens in colombia-2018.pdf

[8] Bhutani S, van Dellen MR, Cooper JA. Longitudinal Weight Gain and Related Risk Behaviors during the COVID-19 Pandemic in Adults in the US. Nutrients. 2021;13(2):671. DOI: https://doi.org/10.3390/ nu13020671

[9] Valenzuela PL, Simpson LJ, Castillo-García A, Lucia A. Physical activity: A coadjuvant treatment to COVID-19 vaccination? Brain Behav Immun. 2021 my.;94:13. DOI: https://doi.org/10.1016/j. bbi.2021.03.003 